\section{(1)}

CrossMark

\title{
From ARIA guidelines to the digital transformation of health in rhinitis and asthma multimorbidity
}

\author{
Jean Bousquet ${ }^{1,2,3,4,5}$, Josep M. Anto ${ }^{6,7,8}$, Claus Bachert ${ }^{9}$, \\ Sinthia Bosnic-Anticevich ${ }^{10}$, Marina Erhola ${ }^{11}$, Tari Haahtela ${ }^{12}$, \\ Peter W. Hellings ${ }^{13,14}$, Piotr Kuna ${ }^{15}$, Oliver Pfaar ${ }^{16}$, Boleslaw Samolinski ${ }^{17}$, \\ Holger J. Schünemann ${ }^{18}$, Aziz Sheikh ${ }^{19}$, Dana Wallace ${ }^{20}$, and the ARIA \\ study group
}

\begin{abstract}
Affiliations: ${ }^{1}$ University Hospital Montpellier, Montpellier, France. ${ }^{2}$ MACVIA-France, Fondation partenariale FMC VIA-LR, Montpellier, France. ${ }^{3}$ INSERM U 1168, VIMA: Ageing and chronic diseases, Epidemiological and public health approaches, Villejuif, France. "Université Versailles St-Quentin-en-Yvelines, UMR-S 1168, Montigny le Bretonneux, France. ${ }^{5}$ Charité, Universitätsmedizin Berlin, Humboldt-Universität zu Berlin, and Berlin Institute of Health, Comprehensive Allergy Center, Dept of Dermatology and Allergy, Berlin, Germany. ${ }^{6}$ ISGlobAL, Centre for Research in Environmental Epidemiology (CREAL), Barcelona, Spain. ${ }^{7}$ Universitat Pompeu Fabra (UPF), Barcelona, Spain. ${ }^{8} \mathrm{CIBER}$ Epidemiología y Salud Pública (CIBERESP), Barcelona, Spain. ${ }^{9}$ Upper Airways Research Laboratory, ENT Dept, Ghent University Hospital, Ghent, Belgium. ${ }^{10}$ Woolcock Institute of Medical Research, University of Sydney and Woolcock Emphysema Centre and Sydney Local Health District, Glebe, Australia. ${ }^{11}$ National Insitute for Health and Welfare, Helsinki, Finland. ${ }^{12}$ Skin and Allergy Hospital, Helsinki University Hospital, and University of Helsinki, Helsinki, Finland. ${ }^{13}$ Dept of Otorhinolaryngology, University Hospitals Leuven, Leuven, Belgium. ${ }^{14}$ Academic Medical Center, University of Amsterdam, Amsterdam, The Netherlands. ${ }^{15}$ Division of Internal Medicine, Asthma and Allergy, Barlicki University Hospital, Medical University of Lodz, Lodz, Poland. ${ }^{16}$ Dept of Otorhinolaryngology, Head and Neck Surgery, Section of Rhinology and Allergy, University Hospital Marburg, Phillipps-Universität Marburg, Marburg, Germany. ${ }^{17}$ Dept of Prevention of Envinronmental Hazards and Allergology, Medical University of Warsaw, Warsaw, Poland. ${ }^{18}$ Dept of Health Research Methods, Evidence, and Impact, Division of Immunology and Allergy, Dept of Medicine, McMaster University, Hamilton, ON, Canada. ${ }^{19}$ The Usher Institute of Population Health Sciences and Informatics, The University of Edinburgh, Edinburgh, UK. ${ }^{20}$ Nova Southeastern University, Fort Lauderdale, FL, USA.
\end{abstract}

Correspondence: Jean Bousquet, CHU Arnaud de Villeneuve, 371 Avenue du Doyen Gaston Giraud, 34295 Montpellier Cedex 5, France. E-mail: jean.bousquetवorange.fr

@ERSpublications

ARIA has evolved, with strong political commitment, from the first multimorbidity guideline in respiratory diseases to an exemplar for the future digital transformation of health and care for the management of patients with long-term conditions http://bit.ly/35fBlhN

Cite this article as: Bousquet J, Anto JM, Bachert C, et al. From ARIA guidelines to the digital transformation of health in rhinitis and asthma multimorbidity. Eur Respir J 2019; 54: 1901023 [https://doi. org/10.1183/13993003.01023-2019].

\section{Introduction}

In all societies, the disease burden and the healthcare costs for people with allergic and chronic respiratory diseases are increasing rapidly [1]. Most economies are struggling to deliver consistent high-quality healthcare. There is a need to support the transformation of the healthcare system for integrated care through leveraging developments in digital health [2]. The term "digital health" refers to advanced medical technologies, disruptive innovations and digital communication tools aiming to provide best healthcare practice [3]. 
As a proof-of-concept for chronic disease care, MASK (Mobile Airways Sentinel NetworK), a new project of the ARIA (Allergic Rhinitis and its Impact on Asthma) initiative [4], and POLLAR (Impact of Air POLLution on Asthma and Rhinitis, EIT Health) [5], in collaboration with professional and patient organisations in the field of allergy and airway diseases, are proposing real-life integrated care pathways (ICPs) centred around the patient with rhinitis and asthma multimorbidity. These next-generation ICPs represent a prism through which the potential for digitally transformed healthcare can be viewed.

The European Union (EU) and global political agendas have been of considerable importance in advancing this project and MASK has been recognised by DG Santé (European Commission Directorate-General for Health and Food Safety) as an example of good practice in the field of digitally enabled, integrated, person-centred care [6].

ARIA has followed several steps from a guideline to a digital solution for care pathways centred around the patient. These steps are depicted in this editorial.

\section{Digital transformation of health and care in the European Union}

The 2017 report on the state of health in the EU (State of Health in the EU "Companion Report 2017"; https://ec.europa.eu/health/state) proposed a rethinking of health and care systems in order to ensure their sustainability and their ability to enhance health promotion as well as to provide a patient-centred care meeting citizens' needs (https://ec.europa.eu/transparency/regdoc/rep/1/2018/FR/COM-2018-233-F1-FRMAIN-PART-1.PDF).

Digital solutions for health and care can increase the well-being of millions of citizens and radically change services. In its mid-term review on the implementation of the digital single market strategy (https://ec.europa.eu/transparency/regdoc/rep/1/2017/EN/COM-2017-228-F1-EN-MAIN-PART-1.PDF), the EU Commission proposed digital solutions, such as wearables and mHealth Apps, in which citizens can actively engage in health promotion and self-management of chronic diseases [7]. Digital tools offer a vehicle through which scientific knowledge can be disseminated in an easily accessible form. Digital solutions can be used across all medical and non-medical sectors to promote evidence-based information and awareness campaigns on healthy lifestyles. Digital tools also enable citizens to provide their doctors with feedback and data on their health.

\section{ARIA}

ARIA was initiated during a World Health Organization workshop in 1999 [8] and has evolved in five phases. These are summarised below.

Phase 1 (1999)

Development and update of an evidence-based document to provide a guide for the diagnosis and management of allergic rhinitis and asthma multimorbidity [8,9]. ARIA has been disseminated widely: it is implemented in over 80 countries and has been translated into over 50 languages [10].

Phase 2 (2010)

ARIA was revised using the GRADE (Grading of Recommendation, Assessment, Development and Evaluation) approach for assessing the strength of evidence underpinning recommendations [11, 12].

Phase 3 (2016)

Implementation of mHealth tools for individualised and predictive medicine to develop ICPs for the management of allergic rhinitis and asthma by a multidisciplinary team centred around the needs of patients (MASK) $[4,5,13]$.

Phase 4 (2018)

Digital transformation of health and care [6] as well as change management to improve health and provide well-being for allergic rhinitis and asthma sufferers across their life cycle, irrespective of gender, age, or socio-economic status and with the overarching aim to reduce health and social inequities [14].

Phase 5 (2020, ARIA planetary health)

Digital transformation of health and care to sustain planetary health.

\section{Next-generation care pathways in rhinitis and asthma multimorbidity}

There is a need to support the digital transformation of health and care with ICPs. ICPs are structured multidisciplinary care plans detailing the key steps of patient care [15]. They promote the translation of guideline recommendations into local protocols and their application to clinical practice. ICPs have been 
proposed with a focus on mHealth technologies that should enhance self-management and adherence to guidelines and ICPs.

An innovative patient-centred approach for ICPs has been proposed by the ARIA expert group for rhinitis and asthma multimorbidity to be scaled up to chronic diseases. Five aspects of ICPs have been developed.

Patient participation, health literacy and self-care through technology-assisted patient and public engagement and involvement

Real-life observational studies, such as MASK, have shown that a large number of allergic rhinitis patients self-medicate using over-the-counter drugs $[16,17]$. However, this is not reflected in guidelines. In order to improve day-to-day decisions about allergic rhinitis management, it is important to consider self-management support, and patient and public engagement and involvement (PPE/I). Self-management support is a key principle for ICPs in chronic diseases. It is the assistance that professionals (pharmacy, primary care, specialist), patient's organisations and other sources, as well as informal caregivers, give to patients in order to make decisions about their condition, to manage disease and health-related tasks [18] and to support shared-decision making. PPE/I, defined as the "knowledge, skills and confidence a person has in managing his/her own health and healthcare" [19], is a goal of many ICP models. mHealth, such as apps running on consumer smart devices, has the potential to profoundly impact PPE/I.

\section{ICP implementation by pharmacists}

Pharmacists are trusted healthcare professionals. The many patients who use over-the-counter medications are seen by the pharmacist, who is the initial point of contact for allergic rhinitis management in most countries. The role of the pharmacist in allergic rhinitis ICPs is important. The ARIA-pharmacy ICP includes a diagnostic questionnaire specifically focusing attention on key symptoms and markers of the disease, a systematic diagnosis guide (including differential diagnoses) and a simple flowchart with proposed treatment for allergic rhinitis and asthma multimorbidity. Key prompts for referral within the ICP are included. However, the ARIA-pharmacy ICP should be adapted to local healthcare environments and situations, as regional (national) differences exist in pharmacy care [20].

\section{Next-generation ARIA guidelines}

The next generation of guidelines assessed GRADE recommendations in allergic rhinitis and asthma using real-world evidence. Randomised controlled trials on treatment effects are included, as well as real-world data obtained by mHealth tools, including MASK. The aim is to confirm effectiveness or refine current recommendations [21]. MASK results were used to refine GRADE guidelines [16]. Adherence to treatment is very low; less than $5 \%$ of users record symptoms and medications for a period of 2 weeks [22]. The app is based on unsupervised input of data and there may be a bias related to potentially missing data input. It is therefore important to further test whether on-demand treatment is equally or even more efficient than regular continuous treatment. Moreover, guidelines should consider both regular and on-demand treatment $[16,22,23]$. Patients do not typically follow guidelines or physicians' prescriptions and often self-medicate depending on the control of their symptoms [16, 17]. They frequently change the medications they use, preferring an effective medication with a rapid onset of action. Often, patients increase their medications but do not get a better control [17]. As in resistant hypertension, and as defined by the number of medications used to control the disease [24], many patients are uncontrolled despite using many medications, extending the SCUAD (severe chronic upper airway disease) concept to resistant rhinitis [25]. Patients who are controlled tend to stop their medications rapidly. Thus, it is essential to produce next-generation ARIA guidelines that include real-world evidence embedding behavioural science and to consider patients' needs and satisfaction.

\section{ARIA ICPs for allergen immunotherapy}

Allergen immunotherapy (AIT) is a proven therapeutic option for allergic rhinitis and asthma. Many guidelines or national practice guidelines have been produced. However, the approach to including evidence varies; many are complex and none propose ICPs. This project reviewed ICPs for AIT using strict criteria and provided simple recommendations by healthcare professionals and other stakeholders [26]. The decision to prescribe AIT should be individualised and based on the relevance of the allergens, the persistence of symptoms despite appropriate medications, and on the availability of good-quality and efficacious extracts. AIT is selected by specialists for stratified patients. There are no currently available validated biomarkers that can predict AIT success. In adolescents and adults, AIT should, in general, be reserved for patients with moderate/severe allergic rhinitis or for those with moderate asthma who, despite appropriate pharmacotherapy and adherence, continue to exhibit exacerbations that appear to be related to allergen exposure. AIT may be even more advantageous in patients with multimorbidity. In children with 
rhinitis, AIT may prevent the onset of asthma. mHealth tools offer the potential for stratification and efficient follow-up of patients.

\section{Embedding air pollution, aerobiology and novel approaches in ICPS}

Although environmental factors play a major role in allergic diseases, no guideline or recommendation has ever included them. Based on the results of POLLAR [5], ARIA next-generation ICPs will incorporate exposure to environmental factors such as pollen and air pollution, as well as novel approaches such as artificial intelligence that have not been considered up to now. As there is increasing evidence that patients' choices and behaviours have an impact on the planet, this will be the background of ARIA Planetary Health [27].

\section{Digital transformation of health in rhinitis and asthma}

In the context of implementing communication on the digital transformation of health and care, DG Santé, in collaboration with the newly established EU Commission Expert Group "Steering Group on Health Promotion, Disease Prevention and Management of Non-Communicable Diseases" (https://ec. europa.eu/health/non_communicable_diseases/steeringgroup_promotionprevention_en), supported the scaling up and wider implementation of good practices in the field of digitally enabled, integrated, person-centred care. MASK was one of the nine selected good practice initiatives [6].

The goals of ARIA phase 4 are in line with those of EIT Health (European Institute for Innovations and Technology) and are developed with high-level collaborations and EU grants (VIGOUR, DigitalHealthEurope, Euriphi and Gatekeeper).

\section{The ARIA political agenda of change management}

The EU political agenda is of great importance in supporting the digital transformation of health and care. AIRWAYS-ICPs (Integrated care pathways for airway diseases) [15], initiated in 2014 by the European Innovation Partnership on Active and Healthy Ageing (DG Santé and DG CONNECT, the Directorate-General for Communications Networks, Content and Technology) [28], launched a collaboration to develop multi-sectoral ICPs. It was a GARD (Global Alliance against Chronic Respiratory Diseases) demonstration project [29]. An EU Summit in Vilnius, Lithuania (2018) proposed multi-sectoral ICPs embedding guided self-management [30]. MASK is a recognised good practice initiative of DG Santé [6].

\section{Conclusions}

ARIA has evolved from the first multimorbidity guideline in respiratory diseases to an example for the digital transformation of health and care. However, there are many hurdles still to overcome to deploy this change management strategy globally [14]. These are worth pursuing as they offer the opportunity to improve outcomes for patients living with one of the most prevalent long-term conditions. They will serve as a basis for improving care provision and health outcomes for people living with other long-term conditions.

The value of real-world data in research has been demonstrated by MASK for a better understanding of phenotypes, adherence and assessment of patient's behaviour. These studies suggest their potential in other respiratory fields, such as COPD or interstitial lung disease, for implementation of guidelines and to obtain real-time information on patient adherence.

Conflict of interest: J. Bousquet reports personal fees from Chiesi, Cipla, Hikma, Menarini, Mundipharma, Mylan, Novartis, Sanofi-Aventis, Takeda, Teva, Uriach (for membership of advisory boards, consultations and honoraria for meeting lectures), and other from KYomed-Innov (shares), outside the submitted work. J.M. Anto has nothing to disclose. C. Bachert reports consultancy fees from Mylan, Stallergenes, ALK, GSK, Sanofi, Astra-Zeneca and Novartis, outside the submitted work. S. Bosnic-Anticevich reports grants from TEVA (unrestricted research grant on inhaler technique), personal fees from TEVA (lecture and advisory board), personal fees from AstraZeneca (lecture and advisory board), personal fees from Boehringer Ingelheim (lecture and advisory board), personal fees from GSK (lecture), personal fees from Sanofi (consultancy), and personal fees from Mylan (consultancy), outside the submitted work. M. Erhola has nothing to disclose. T. Haahtela reports personal fees from Mundipharma and OrionPharma (lecturing fees), outside the submitted work. P.W. Hellings has nothing to disclose. P. Kuna has nothing to disclose. O. Pfaar reports grants and personal fees from ALK-Abelló, Allergopharma, Stallergenes Greer, HAL Allergy Holding B.V./HAL Allergie GmbH, Bencard Allergie GmbH/Allergy Therapeutics, Lofarma, ASIT Biotech Tools S.A., Laboratorios LETI/LETI Pharma and Anergis S.A., grants from Biomay, Nuvo, Circassia and Glaxo Smith Kline, and personal fees from MEDA Pharma/MYLAN, Mobile Chamber Experts (a GA ${ }^{2} L E N$ Partner), Indoor Biotechnologies and Astellas Pharma Global, outside the submitted work. B. Samolinski has nothing to disclose. H.J. Schünemann has nothing to disclose. A. Sheikh reports that he has served as a member of the BTS/SIGN Asthma Guideline and the EAACI Allergen Immunotherapy Guidelines and is currently a member of the GINA Scientific Board. D. Wallace is co-chair of the Joint Task Force on Practice Parameters of the AAAAI/ACAAI. 
Support statement: Funding was received from Fondation FMC VIA-LR. Funding information for this article has been deposited with the Crossref Funder Registry.

\section{References}

1 Zuberbier T, Lotvall J, Simoens S, et al. Economic burden of inadequate management of allergic diseases in the European Union: a GA²LEN review. Allergy 2014; 69: 1275-1279.

2 Gopal G, Suter-Crazzolara C, Toldo L, et al. Digital transformation in healthcare - architectures of present and future information technologies. Clin Chem Lab Med 2019; 57: 328-335.

3 Mesko B, Drobni Z, Benyei E, et al. Digital health is a cultural transformation of traditional healthcare. Mhealth 2017; 3: 38.

4 Bousquet J, Arnavielhe S, Bedbrook A, et al. MASK 2017: ARIA digitally-enabled, integrated, person-centred care for rhinitis and asthma multimorbidity using real-world-evidence. Clin Transl Allergy 2018; 8: 45.

5 Bousquet J, Anto JM, Annesi-Maesano I, et al. POLLAR: Impact of air POLLution on Asthma and Rhinitis; a European Institute of Innovation and Technology Health (EIT Health) project. Clin Transl Allergy 2018; 8: 36.

6 Bousquet J, Bedbrook A, Czarlewski W, et al. Guidance to 2018 good practice: ARIA digitally-enabled, integrated, person-centred care for rhinitis and asthma. Clin Transl Allergy 2019; 9: 16.

7 Downes E, Horigan A, Teixeira P. The transformation of health care for patients: Information and communication technology, digiceuticals, and digitally enabled care. J Am Assoc Nurse Pract 2019; 31: 156-161.

8 Bousquet J, Van Cauwenberge P, Khaltaev N. Allergic rhinitis and its impact on asthma. J Allergy Clin Immunol 2001; 108: Suppl. 5, S147-S334.

9 Bousquet J, Khaltaev N, Cruz AA, et al. Allergic Rhinitis and its Impact on Asthma (ARIA) 2008 update (in collaboration with the World Health Organization, GA ${ }^{2}$ LEN and AllerGen). Allergy 2008; 63: Suppl. 86, 8-160.

10 Bousquet J, Schunemann HJ, Samolinski B, et al. Allergic Rhinitis and its Impact on Asthma (ARIA): achievements in 10 years and future needs. J Allergy Clin Immunol 2012; 130: 1049-1062.

11 Brozek JL, Bousquet J, Baena-Cagnani CE, et al. Allergic Rhinitis and its Impact on Asthma (ARIA) guidelines: 2010 revision. J Allergy Clin Immunol 2010; 126: 466-476.

12 Brozek JL, Bousquet J, Agache I, et al. Allergic Rhinitis and its Impact on Asthma (ARIA) Guidelines - 2016 Revision. J Allergy Clin Immunol 2017; 140: 950-958.

13 Bousquet J, Hellings PW, Agache I, et al. ARIA 2016: Care pathways implementing emerging technologies for predictive medicine in rhinitis and asthma across the life cycle. Clin Transl Allergy 2016; 6: 47.

14 Bousquet J, Hellings PW, Agache I, et al. Allergic Rhinitis and its Impact on Asthma (ARIA) Phase 4 (2018): Change management in allergic rhinitis and asthma multimorbidity using mobile technology. J Allergy Clin Immunol 2019; 143: 864-879.

15 Bousquet J, Addis A, Adcock I, et al. Integrated care pathways for airway diseases (AIRWAYS-ICPs). Eur Respir J 2014; 44: 304-323.

16 Bousquet J, Devillier P, Arnavielhe S, et al. Treatment of allergic rhinitis using mobile technology with real-world data: The MASK observational pilot study. Allergy 2018; 73: 1763-1774.

17 Bedard A, Basagana X, Anto JM, et al. Mobile technology offers novel insights on control and treatment of allergic rhinitis. The MASK study. J Allergy Clin Immunol 2019; 144: 135-143.e6.

18 Da Silva D. Helping People Help Themselves: a Review of the Evidence Considering Whether it is Worthwhile to Support Self-management. London, The Health Foundation, 2011. Available from: www.health.org.uk/sites/health/ files/HelpingPeopleHelpThemselves.pdf

19 Hibbard J, Gilburt H. Supporting People to Manage Their Health: an Introduction to Patient Activation. London, King's Fund, 2014. www.kingsfund.org.uk/sites/files/kf/field/field_publication_file/supporting-people-managehealth-patient-activation-may14.pdf

20 Bosnic-Anticevich S, Costa E, Menditto E, et al. ARIA pharmacy 2018 "Allergic rhinitis care pathways for community pharmacy". Allergy 2019; 74: 1219-1236.

21 Schünemann H. All evidence is real world evidence. thebmjopinion 2019. https://blogs.bmj.com/bmj/2019/03/29/ holger-j-schunemann-all-evidence-is-real-world-evidence/

22 Menditto E, Costa E, Midao L, et al. Adherence to treatment in allergic rhinitis using mobile technology. The MASK Study. Clin Exp Allergy 2019; 49: 442-460.

23 Bachert C, Bousquet J, Hellings P. Rapid onset of action and reduced nasal hyperreactivity: new targets in allergic rhinitis management. Clin Transl Allergy 2018; 8: 25.

24 Nagarajan N, Jalal D. Resistant hypertension: diagnosis and management. Adv Chronic Kidney Dis 2019; 26: 99-109.

25 Bousquet J, Bachert C, Canonica GW, et al. Unmet needs in severe chronic upper airway disease (SCUAD). J Allergy Clin Immunol 2009; 124: 428-433.

26 Bousquet J, Pfaar O, Togias A, et al. 2019 ARIA Care pathways for allergen immunotherapy. Allergy 2019; 74: 2087-2102.

27 Whitmee S, Haines A, Beyrer C, et al. Safeguarding human health in the Anthropocene epoch: report of The Rockefeller Foundation - Lancet Commission on planetary health. Lancet 2015; 386: 1973-2028.

28 Bousquet J, Michel J, Strandberg T, et al. The European Innovation Partnership on Active and Healthy Ageing: the European Geriatric Medicine introduces the EIP on AHA Column. Eur Geriatric Med 2014; 5: 361-362.

29 Bousquet J, Dahl R, Khaltaev N. Global alliance against chronic respiratory diseases. Allergy 2007; 62: 216-223.

30 Valiulis A, Bousquet J, Veryga A, et al. Vilnius declaration on chronic respiratory diseases: multisectoral care pathways embedding guided self-management, mHealth and air pollution in chronic respiratory diseases. Clin Transl Allergy 2019; 9: 7 . 